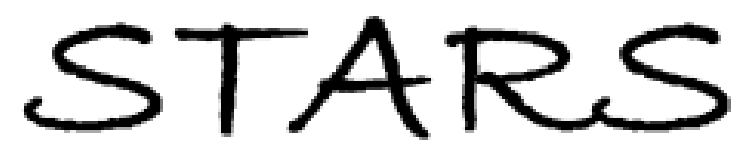

University of Central Florida

STARS

$1-1-2014$

\title{
On the role of long range interactions for the adsorption of sexithiophene on $\mathrm{Ag}(110)$ surface
}

\author{
Jeronimo Matos \\ University of Central Florida \\ Tomas Rojas \\ University of Central Florida \\ Handan Yildirim \\ University of Central Florida \\ Abdelkader Kara \\ University of Central Florida
}

Find similar works at: https://stars.library.ucf.edu/facultybib2010

University of Central Florida Libraries http://library.ucf.edu

This Article is brought to you for free and open access by the Faculty Bibliography at STARS. It has been accepted for inclusion in Faculty Bibliography 2010 s by an authorized administrator of STARS. For more information, please contactSTARS@ucf.edu.

\section{Recommended Citation}

Matos, Jeronimo; Rojas, Tomas; Yildirim, Handan; and Kara, Abdelkader, "On the role of long range interactions for the adsorption of sexithiophene on $\mathrm{Ag}(110)$ surface" (2014). Faculty Bibliography 2010 s. 5806.

https://stars.library.ucf.edu/facultybib2010/5806

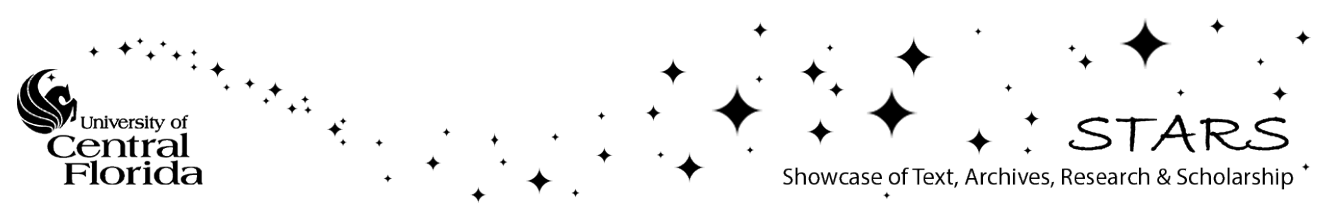




\section{On the role of long range interactions for the adsorption of sexithiophene on Ag(110) surface}

Cite as: J. Chem. Phys. 140, 144703 (2014); https://doi.org/10.1063/1.4870459

Submitted: 18 January 2014 . Accepted: 24 March 2014 . Published Online: 10 April 2014

Jeronimo Matos, Tomas Rojas, Handan Yildirim, and Abdelkader Kara

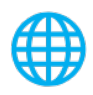

\section{ARTICLES YOU MAY BE INTERESTED IN}

The role of van der Waals forces in water adsorption on metals

The Journal of Chemical Physics 138, 024708 (2013); https://doi.org/10.1063/1.4773901

Perspective: Advances and challenges in treating van der Waals dispersion forces in density functional theory

The Journal of Chemical Physics 137, 120901 (2012); https://doi.org/10.1063/1.4754130

A consistent and accurate ab initio parametrization of density functional dispersion correction (DFT-D) for the 94 elements H-Pu

The Journal of Chemical Physics 132, 154104 (2010); https://doi.org/10.1063/1.3382344

Where in the world is AIP Publishing? Find out where we are exhibiting next 


\title{
On the role of long range interactions for the adsorption of sexithiophene on $\mathrm{Ag}(110)$ surface
}

\author{
Jeronimo Matos, Tomas Rojas, Handan Yildirim, ${ }^{a), b)}$ and Abdelkader Kara ${ }^{\text {b) }}$ \\ Department of Physics, University of Central Florida, Orlando, Florida, 32816, USA
}

(Received 18 January 2014; accepted 24 March 2014; published online 10 April 2014)

\begin{abstract}
The adsorption characteristics of the sexithiophene (6T) molecule on $\operatorname{Ag}(110)$ are studied using density functional theory with the inclusion of van der Waals ( $\mathrm{vdW}$ ) interactions. The stable adsorption configurations on 6T on $\mathrm{Ag}(110)$ as well as the nature of bonding the $\mathrm{Ag}$ substrate are evaluated. We also assess the performance of the vdW-DF method in describing the adsorption, energetics, heights, as well as the interface characteristics with the $\operatorname{Ag}(110)$ surface. We find two lowest adsorption energy configurations, at which the $6 \mathrm{~T}$ molecule aligns with its molecular long axis parallel and perpendicular to the [001] direction, to be energetically close to each other, suggesting that they may coexist. Our findings indicate a significant increase in the $6 \mathrm{~T}$ adsorption energies upon the inclusion of vdW interactions with the highest increase obtained using the opt-type functionals, in particular with the optB86b-vdW functional. The revPBE-vdW and rPW86-vdW2 functionals lead to less enhancement in adsorption energies that is attributed to the strong repulsive nature of these functionals, in agreement with earlier predictions. Upon adsorption of the 6T molecule, the changes in the atomic and electronic structures of the $6 \mathrm{~T}$ molecule and $\mathrm{Ag}$ surface are found to be negligible; there is no charge transfer, and no interface state is observed. The work function is reduced upon adsorption with the largest change is $\sim 0.6 \mathrm{eV}$ obtained using the optB88-vdW functional. The results are in good agreement with the available experimental observations of the adsorption configurations and the work function changes. Based on our results, we conclude that the nature of bonding for 6T on $\mathrm{Ag}(110)$ can be classified as strong physisorption. () 2014 AIP Publishing LLC. [http://dx.doi.org/10.1063/1.4870459]
\end{abstract}

\section{INTRODUCTION}

The organic molecular semiconductors have attracted increasing interest for their potential applications in inexpensive and flexible electronic devices. ${ }^{1,2}$ The viability of these materials to function in place of their inorganic relatives was demonstrated by Lin et al. with the fabrication of a pentacenebased organic thin film transistor (OTFT) shown to achieve field-effect mobility and sub-threshold slope comparable to alpha-Si:H TFTs. ${ }^{3}$ Other studies paid attention instead to oligothiophenes due to their structural diversity and tunability that is suitable for applications such as bio sensing, ${ }^{4,5}$ light emitting diodes, ${ }^{6}$ solar cells, ${ }^{7}$ and field effect transistors. ${ }^{8}$ Among them, sexithiophene $\left(\mathrm{C}_{24} \mathrm{H}_{14} \mathrm{~S}_{6}\right)$ is shown to have a high value of carrier mobility as compared to the other oligothiophenes resulting from its effective pi-conjugation length. ${ }^{9}$ Since the structures of the increasingly miniaturized electronic devices consist of layered thin films each with different compositions and functions, the interface between layers plays a critical role for device performance. For this reason, special attention has been given to better describe the interface characteristics of metal-organic semiconductors.

Several experimental studies have reported the interfacial electronic structure properties of similar systems often using

a) Present address: School of Chemical Engineering, Purdue University, West Lafayette, Indiana 47907, USA.

b) Authors to whom correspondence should be addressed. Electronic addresses: hyildirim@purdue.edu and Abdelkader.Kara@ucf.edu spectroscopic techniques. For instance, ultraviolet photoemission (UV) studies of $6 \mathrm{~T}$ adsorbed on polycrystalline Au and $\mathrm{Ag}$ reported interface dipoles of $1.2 \mathrm{eV}^{10}$ and $0.7 \mathrm{eV},{ }^{11}$ respectively. In these studies, the interface dipoles were estimated from the change in the work functions upon molecular adsorption. The Photo-Emission Electron Microscopy (PEEM) study, by Wagner et al., ${ }^{12}$ of $6 \mathrm{~T}$ adsorption on $\operatorname{Ag}(110)$ reported an increase in the emission probability of their sample during the deposition of the first 6T monolayer, which they attributed to a reduction in the work function. In another study, using x-ray photoelectron spectroscopy (XPS) and ultraviolet photoelectron spectroscopy (UPS), Amsalem et al. ${ }^{13}$ observed the formation of an interface dipole and charge transfer at the organic-organic interface of $\mathrm{C}_{60}$ deposited over a thin layer of $6 \mathrm{~T} / \mathrm{Ag}(111)$.

The bonding nature between a molecule and the metal support affects the growth characteristics and the ordering of molecular thin films on surfaces as the mobility and self-organization are controlled by molecule-substrate and molecule-molecule interactions. ${ }^{14,15}$ For the case of 6T, studies by Yoshikawa et al. ${ }^{9}$ and Kiguchi et al. ${ }^{16}$ of 6T grown on $\mathrm{Cu}(110)$ and $\mathrm{Ag}(110)$, respectively, using NEXAFS (Near Edge X-Ray Absorption Fine Structure), reported that, after annealing, the $6 \mathrm{~T}$ molecule aligns with its molecular long axis parallel to the [001] direction. Furthermore, the authors reported that 6T molecules adsorb with a narrower alignment distribution on $\mathrm{Ag}(110)$, as compared with $\mathrm{Cu}(110)$, and attributed the observation to the difference in the mismatch 
between the molecular ring-ring distance and the interatomic distance along the long bridge for the two substrates. ${ }^{16}$ In contrast, Wagner et al., ${ }^{12}$ based on scanning tunneling microscopy (STM) measurements, reported that the first layer of 6T adsorbed on $\mathrm{Ag}(110)$ consists of a mixture of molecular alignment orientations, parallel and perpendicular to the [001] direction. Prato et al., using helium atom scattering (HAS), low-energy electron diffraction (LEED), and STM reported molecular long axis alignment in the [110] direction for 6T adsorbed on $\mathrm{Au}(110) .{ }^{17}$

First principles calculations based on the density functional theory (DFT) ${ }^{18,19}$ are helpful for bridging the gap between the experimental reports on molecular orientations, binding energies and heights, as well as the electronic structure characteristics of the metal-semiconductor interface. ${ }^{20}$ However, the accuracy of the calculations largely depends on the appropriate choice of the exchange-correlation term, which accurately captures the interaction characteristics at the interface. It is now established that standard DFT calculations with the exchange-correlation functionals such as local density approximation (LDA) and generalized gradient approximation (GGA) cannot describe the long-range interactions that play an important role in the interaction between organic molecules and metal substrates. ${ }^{21-27}$ We have witnessed increasing efforts in recent years to construct van der Waals (vdW) functionals that include these interactions, and many studies reported the improvement in the description of interface properties with the inclusion of vdW interactions, see the discussions in the review articles. ${ }^{28-31}$ Having said that, the performance of these functionals regarding their transferability between many systems with varying nature of interaction, the functional groups in organic molecules, and varying surface coordination and type have not yet been well tested to help in further improvements. Nowadays, we are witnessing such efforts in this direction and these efforts are without a question, beneficial for obtaining insights into the performance of these functionals on a variety of interaction regimes and types. $^{32-35}$

The main focus of our paper is to study the adsorption characteristics of the 6T molecule on $\mathrm{Ag}(110)$ utilizing DFT with the inclusion of $\mathrm{vdW}$ interactions to obtain insights into the preferred adsorption configurations, and interface characteristics with the Ag substrate for evaluating the nature of bonding. Concurrently, we examine the energetics and adsorption heights dependence on the few vdW functionals, and their performance with respect to the GGA functional of Perdew, Burke and Ernzerhof (PBE). ${ }^{36,37}$ In the present study, we focus on the vdW-DF family of functionals, which belong to the third level in the "stairway to heaven" for vdWs as described in the review article by Klimes et al., ${ }^{38}$ and compare the trends we find with those obtained in our earlier study for benzene and olympicene radical on metal surfaces. ${ }^{32,33}$ Using the vdW functionals, namely, optPBE-vdW, ${ }^{24}$ optB88-vdW, ${ }^{24}$ optB86b-vdW, ${ }^{39}$ revPBE-vdW, ${ }^{21,40}$ and rPW86-vdW2, ${ }^{41,42}$ we calculate the adsorption energies, heights, electronic structure characteristics of the interface, the change in work function, and the charge transfer. The details regarding the differences between these vdW functionals have been discussed in the review article, ${ }^{38}$ and in our recent paper for benzene adsorption on several metal surfaces, ${ }^{32}$ and hence omitted here.

Our study shows that the lowest adsorption energy configuration is the one at which the molecule's long axis is parallel to the [001] direction. The adsorption energies are enhanced with vdW interactions, and the degree of enhancement can vary significantly depending on the functionals. Increase in adsorption energies is accompanied with decrease in adsorption heights for the opt-type functionals as compared to those of PBE, while the adsorption heights increase with the revPBE-vdW and rPW86-vdW2 functionals. The latter is attributed to a strong repulsive nature of these functionals at short ranges. The electronic structure analysis suggests no charge transfer or formation of an interface state upon adsorption as well as no significant structural change of the molecule and the surface. The calculations suggest a reduction of the work function upon adsorption of the molecule in agreement with the experimental findings.

In Sec. II, we provide a concise description of the computational details and adsorption configurations, following that in Sec. III, the change in structural characteristics, the adsorption energetics, and heights, and the electronic structure changes are discussed within the context of the role of $\mathrm{vdW}$ interactions, and the performances of these $\mathrm{vdW}$ functionals. Finally, in Sec. IV, we summarize our results.

\section{COMPUTATIONAL DETAILS}

The calculations are carried out within the DFT framework using VASP code (version 5.3.3). ${ }^{43-45}$ For assessing the role of $\mathrm{vdW}$ interactions on the adsorption characteristics, optB88-vdW, optB86b-vdW, optPBE-vdW, revPBE$\mathrm{vdW}$, and rPW86-vdW2 functionals are used, and comparisons are made with the results of the GGA-PBE exchange correlation functional. The interaction between the valence electrons and ionic cores is described by the projector augmented wave (PAW) method. ${ }^{46,47}$ The plane wave energy cutoff is set to $400 \mathrm{eV}$, and the Brillouin zone is sampled with (12 $\times 3 \times 1)$ and $(6 \times 2 \times 1) \mathrm{k}$-point meshes for the molecular orientation parallel to the [110] and [001], respectively.

The supercell models with dimensions $(2 \times 10)$ and $(4$ $\times 7)$ are constructed with a 5 layers slab, each layer containing either 20 or $28 \mathrm{Ag}$ atoms depending on the molecular orientation considered with respect to the Ag surface. A $20 \AA$ vacuum region is used to separate the two surfaces. Upon adsorption of the 6T molecule, the bottom two layers are fixed during the structural optimization with a force criterion of $0.01 \mathrm{eV} / \AA$. For each adsorption configuration, the structural optimization is performed for the 6T molecule alone, Ag substrate alone, and 6T on Ag substrate systems. The lattice constants for $\mathrm{Ag}$ are calculated for all considered vdW functionals, and found to be in good agreement with previously reported theoretical values; ${ }^{32,39} 4.175 \AA$ (PBE), $4.328 \AA$ (optB86b-vdW), $4.258 \AA$ (optB88-vdW), $4.179 \AA$ (optPBEvdW), $4.108 \AA$ (revPBE-vdW), and $4.146 \AA$ (rPW86-vdW2).

We consider two adsorption orientations for the $6 \mathrm{~T}$ molecule on $\operatorname{Ag}(110)$ as shown in Figure 1, with the molecular axis parallel to either the [110] or [001] directions as discussed in the previous literature. ${ }^{9,12,16,17}$ For each 


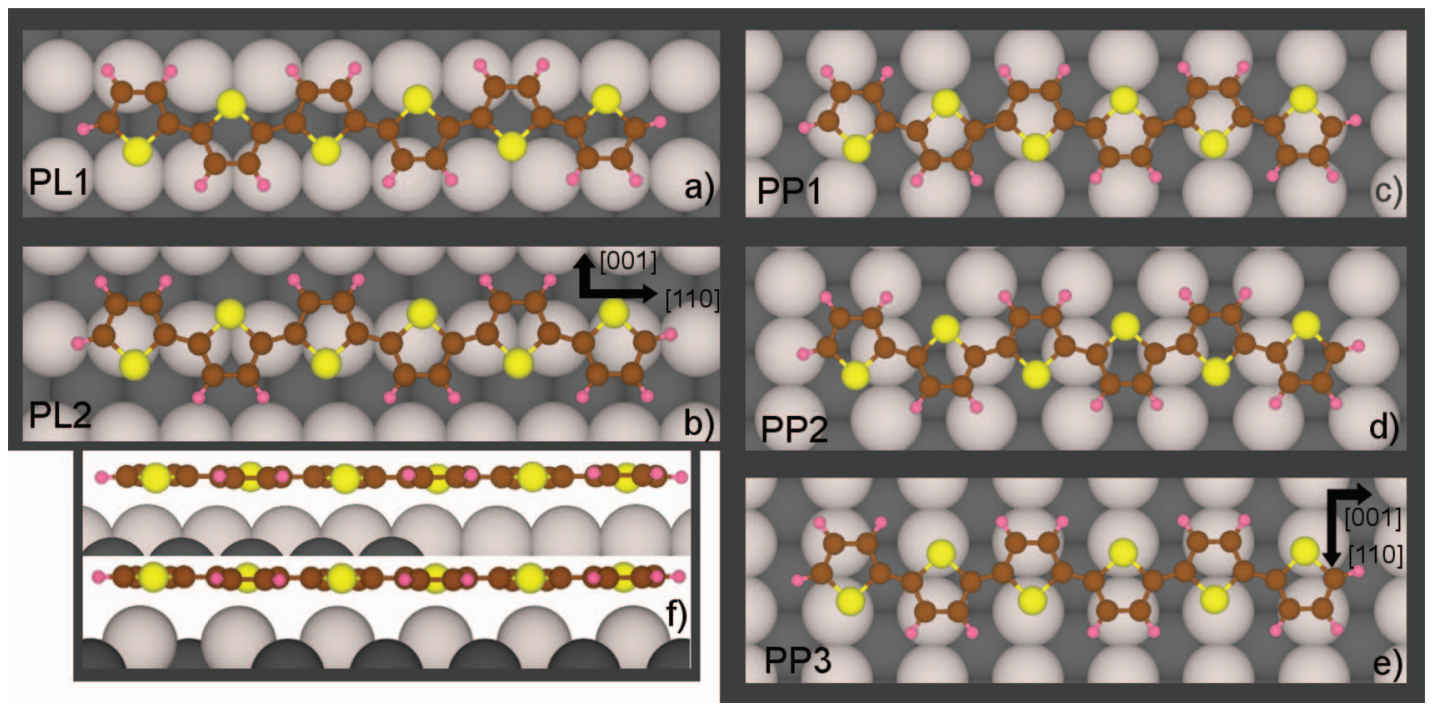

FIG. 1. Schematic top views of the optimized adsorption configurations of 6T on $\mathrm{Ag}$ (110) using optB86b-vdW functional for (a) PL1, (b) PL2, (c) PP1, (d) PP2, (e) PP3, and (f) side views of PL1 and PP3 adsorption configurations. The light gray, dark gray, brown, pink, and yellow spheres represent Ag atoms in the 1st layer, $\mathrm{Ag}$ atoms in the 2nd layer, $\mathrm{C}$ atoms, $\mathrm{H}$ atoms, and $\mathrm{S}$ atoms, respectively.

orientation considered, the 6T molecule is adsorbed at different adsorption sites whose surroundings are unique, and can be defined with the position of $\mathrm{S}$ atoms in the molecule. For the case at which $6 \mathrm{~T}$ is adsorbed on the Ag surface parallel to the [110] direction, two adsorption configurations are considered, namely PL1 and PL2 (see Figures 1(a) and 1(b)). For the case where 6T is absorbed parallel to the [001] direction, we consider three adsorption configurations, PP1, PP2, and PP3 as shown in Figures 1(c)-1(e). For the PL1 configuration, the 6T molecule is adsorbed on the open channel of $\mathrm{Ag}(110)$ surface, running parallel to the [110] direction, while for the PL2 configuration, 6T is adsorbed on top of the $\mathrm{Ag}$ atoms. For the PP1, PP2, and PP3 configurations, the C-S rings of the 6T molecule are adsorbed on top of $\mathrm{Ag}$ atoms, in between the $\mathrm{Ag}$ atom rows, and at the bridge sites made of two surface $\mathrm{Ag}$ atoms. For all calculations, the $6 \mathrm{~T}$ molecule is brought to a distance of $\sim 3 \AA$ from the surface and structural optimization is performed with no constraint imposed on the system except that the two bottom layers of the surface are fixed. Using six layers slab, we find the change in the $6 \mathrm{~T}$ adsorption energy for PP3 configuration with the optB86b-vdW functional is negligible, only $57 \mathrm{meV}$. For a quantitative analysis of the charge transfer upon adsorption of the 6T molecule on the $\operatorname{Ag}(110)$ surface, we perform the Bader charge analysis. ${ }^{48}$ To calculate the Bader volume and charge associated with each atom, we use the code developed by Henkelman et al. ${ }^{49}$

\section{RESULTS AND DISCUSSION}

\section{A. Adsorption configurations and energies for $6 \mathrm{~T}$ on $\mathrm{Ag}(110)$}

6T adsorption energies on $\operatorname{Ag}(110)$ for all of the considered configurations, calculated using PBE as well as the vdW functionals, are summarized in Table I. For the PL1 adsorption configuration, the molecule's long axis is oriented along the substrate's [110] direction and positioned at the center of the rows made of $\mathrm{Ag}$ atoms (see Fig. 1(a)). After the relaxation, the $\mathrm{S}$ atoms at the two ends as well as the one in the middle of the $6 \mathrm{~T}$ molecule are positioned along the channel off center in the [110] and [001] directions above the top layer Ag atoms, while the second and fifth sulfur atoms are positioned off center in the [001] direction at half of the nearest neighbor distance of the top layer Ag atoms. The PL2 adsorption configuration differs from PL1 by a translation of $6 \mathrm{~T}$ in the [001] direction so that its molecular axis is positioned over the center of the (110) surface row of Ag atoms. Similarly to the PL1 adsorption configuration, the second and the fifth $\mathrm{S}$ atoms are positioned off center in the [001] direction, at half of the nearest neighbor distance between the $\mathrm{Ag}$ atoms underneath the molecule.

By examining the results summarized in Table I on the adsorption energies using PBE functional, we find that the highest adsorption energies on $\operatorname{Ag}(110)$ correspond to the PP3 configuration $(0.87 \mathrm{eV})$, followed by the PL1 configuration $(0.60 \mathrm{eV})$, while the lowest is found for the PP2 configuration $(0.28 \mathrm{eV})$. With the inclusion of $\mathrm{vdW}$ interactions, the results summarized in the Table I clearly indicate enhancement in the adsorption energies from those obtained using PBE. We can see that the highest adsorption energies, considering all of the vdW functionals, correspond to the PP3 and PL1 adsorption configurations, with the former being higher than the latter. One exception to this trend is found for the revPBE-vdW functional, for which the adsorption energy for the PL1 configuration is slightly higher than that of PP3. Note that earlier experimental work by Yoshikawa et al. ${ }^{9}$ reported that, after annealing, the 6T molecule aligns, primarily, with its molecular long axis parallel to the [001] direction on $\operatorname{Ag}(110)$, while another experimental study, by Wagner et al., ${ }^{12}$ showed that the first layer of $6 \mathrm{~T}$ adsorbed on $\mathrm{Ag}(110)$ consists of a mixture of the molecular long axis orientations parallel and perpendicular to the [001] direction. Although our coverage is not as high as those in the experiments, the relatively small difference in adsorption energies of $6 \mathrm{~T}$, with 
TABLE I. Adsorption energies (in eV) and heights (in $\AA$ ) for $6 \mathrm{~T}$ adsorbed on $\mathrm{Ag}(110)$ using PBE, optB86b-vdW, optB88-vdW, optPBE-vdW, revPBE$\mathrm{vdW}$, and rPW86-vdW2 functionals for all considered adsorption configurations. The adsorption energy is calculated as $\mathrm{E}_{\mathrm{ads}}=-\left(\mathrm{E}_{6 \mathrm{~T} / \mathrm{Ag}(110)}-\mathrm{E}_{\mathrm{Ag}(110)}\right.$ $-\mathrm{E}_{6 \mathrm{~T}}$ ), where the subscripts $6 \mathrm{~T} / \mathrm{Ag}(110), \operatorname{Ag}(110)$, and $6 \mathrm{~T}$ refer to the total energies of 6T on the Ag surface, the clean Ag surface, and isolated 6T systems, respectively. The adsorption heights are calculated using the average $\mathrm{z}$ positions of all molecule atoms ( $\left.\mathrm{d}_{\mathrm{ads}} \mathrm{av}\right)$, the average $\mathrm{z}$ positions of the $\mathrm{C}$ atoms $\left(\mathrm{d}_{\mathrm{ads}} \mathrm{CM}\right)$, and the average $\mathrm{z}$ positions of the $\mathrm{S}$ atoms $\left(\mathrm{d}_{\mathrm{SM}}\right)$ from the average $\mathrm{z}$ positions of the metal surface atoms.

\begin{tabular}{|c|c|c|c|c|c|}
\hline Method & Configuration & $\mathrm{E}_{\text {ads }}(\mathrm{eV})$ & $\mathrm{d}_{\text {ads_av }}(\AA)$ & $\mathrm{d}_{\text {ads_CM }}(\AA)$ & $\mathrm{d}_{\mathrm{SM}}(\AA)$ \\
\hline \multirow[t]{5}{*}{ PBE } & PL1 & 0.60 & 3.09 & 3.08 & 3.03 \\
\hline & PL2 & 0.38 & 3.57 & 3.58 & 3.57 \\
\hline & PP1 & 0.43 & 3.46 & 3.46 & 3.26 \\
\hline & PP2 & 0.28 & 3.51 & 3.51 & 3.44 \\
\hline & PP3 & 0.82 & 3.05 & 3.04 & 2.95 \\
\hline \multirow[t]{5}{*}{ optB86b-vdW } & PL1 & 4.02 & 2.75 & 2.73 & 2.68 \\
\hline & PL2 & 3.10 & 2.97 & 2.99 & 3.04 \\
\hline & PP1 & 3.36 & 3.01 & 3.01 & 2.86 \\
\hline & PP2 & 3.36 & 3.00 & 3.00 & 2.86 \\
\hline & PP3 & 4.38 & 2.70 & 2.70 & 2.71 \\
\hline \multirow[t]{5}{*}{ optB88-vdW } & PL1 & 3.77 & 2.87 & 2.86 & 2.81 \\
\hline & PL2 & 3.01 & 3.00 & 3.01 & 3.07 \\
\hline & PP1 & 3.20 & 3.05 & 3.06 & 2.98 \\
\hline & PP2 & 3.12 & 3.03 & 3.03 & 2.98 \\
\hline & PP3 & 4.08 & 2.77 & 2.78 & 2.78 \\
\hline \multirow[t]{5}{*}{ optPBE-vdW } & PL1 & 3.20 & 2.98 & 2.98 & 2.94 \\
\hline & PL2 & 2.71 & 3.11 & 3.13 & 3.24 \\
\hline & PP1 & 2.85 & 3.16 & 3.17 & 3.09 \\
\hline & PP2 & 2.79 & 3.15 & 3.15 & 3.11 \\
\hline & PP3 & 3.47 & 2.91 & 2.91 & 2.90 \\
\hline \multirow[t]{5}{*}{ revPBE-vdW } & PL1 & 2.31 & 3.25 & 3.25 & 3.21 \\
\hline & PL2 & 2.09 & 3.32 & 3.34 & 3.51 \\
\hline & PP1 & 2.13 & 3.45 & 3.46 & 3.39 \\
\hline & PP2 & 2.11 & 3.43 & 3.43 & 3.39 \\
\hline & PP3 & 2.13 & 3.45 & 3.46 & 3.39 \\
\hline \multirow[t]{5}{*}{ rPW86-vdW2 } & PL1 & 2.43 & 3.15 & 3.15 & 3.12 \\
\hline & PL2 & 2.07 & 3.23 & 3.25 & 3.39 \\
\hline & PP1 & 2.12 & 3.34 & 3.35 & 3.26 \\
\hline & PP2 & 2.08 & 3.30 & 3.30 & 3.29 \\
\hline & PP3 & 2.53 & 3.15 & 3.15 & 3.14 \\
\hline
\end{tabular}

its molecular long axis parallel and perpendicular to the [001] direction, suggests that both configurations can coexist. For all configurations, the highest increase in the adsorption energies, from those of the PBE, is found with the opt-type functionals, with the following order in energies $E_{a d s}$ optB86b-vdW $>\mathrm{E}_{\mathrm{ads}}{ }^{\text {optB88-vdW }}>\mathrm{E}_{\mathrm{ads}}{ }^{\text {optPBE-vdW }}$, while the revPBE-vdW and rPW86-vdW2 functionals leading to a smaller increase in the adsorption energies (Figure 2). A similar trend was obtained in our recent work on benzene adsorption on several metal surfaces, ${ }^{32}$ Olympicene radical on $\mathrm{Cu}(111),{ }^{33}$ as well as an earlier study on water adsorption on metal surfaces, using the same vdW functionals. ${ }^{34}$ The revPBE-vdW and rPW86vdW2 functionals have been shown to lead to strong repulsion at short ranges, and most often predict low adsorption energies and large adsorption heights. ${ }^{24,39,50,51}$ We found, in our recent work that these functionals behave differently from the opt-type functionals, and their performance differs significantly for the adsorption on reactive metal substrates. ${ }^{32}$ The highest adsorption energy among all configurations is $4.38 \mathrm{eV}$ for the PP3 configuration and obtained using the optB86bvdW functional.

The PL1 adsorption configuration has a higher binding energy than PL2 by $0.22 \mathrm{eV}$ (PBE), $0.92 \mathrm{eV}$ (optB86b-vdW), $0.76 \mathrm{eV}$ (optB88-vdW), $0.49 \mathrm{eV}$ (optPBE-vdW), $0.22 \mathrm{eV}$ (revPBE-vdW), and $0.36 \mathrm{eV}$ (rPW86-vdW2). We find that the 6T molecule in the PP3 configuration binds more strongly than in PL1 by $0.22 \mathrm{eV}$ (PBE), $0.36 \mathrm{eV}$ (optB86), $0.31 \mathrm{eV}$ (optB88-vdW), $0.28 \mathrm{eV}$ (optPBE-vdW), $0.10 \mathrm{eV}$ (rPW86vdW2), and $0.18 \mathrm{eV}$ (lower) with revPBE-vdW. The similarity between the PL1 and PP3 adsorption configurations is evident from the Fig. 1, at which at least every other ring occupies the bridge and/or slightly off bridge sites.

In the PP1 adsorption configuration, 6T has its molecular long axis parallel to the [001] direction and it is positioned over the top layer Ag atoms in the [001] direction. The separation between the $\mathrm{Ag}$ atoms in the [001] direction is the lattice parameter of $\mathrm{Ag}$ and the distance between the rings in 6T is close to the lattice parameter of Ag. However, the difference is still large enough to not to allow full epitaxy. After
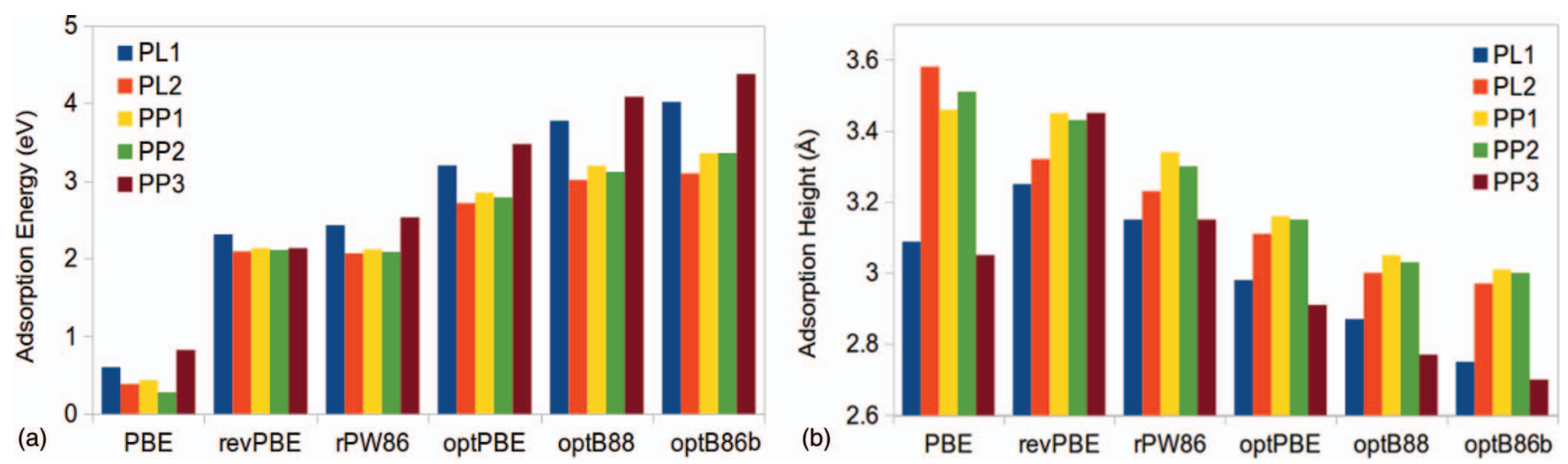

FIG. 2. 6T adsorption characteristics on $\mathrm{Ag}(110)$. (a) Adsorption energies and (b) adsorption heights obtained using PBE, optB86b-vdW, optB88-vdW, optPBE$\mathrm{vdW}$, revPBE-vdW, and rPW86-vdW2 functionals for all considered adsorption configurations. 
relaxation, the molecule does not stretch and the top layer Ag atoms are not shifted from their positions to allow for any epitaxial matching to occur with the adsorbed 6T molecule. One of the $\mathrm{S}$ atoms in the center of the molecule is positioned directly over the top of the short bridge site on $\operatorname{Ag}(110)$, while the rest of the $S$ atoms in the molecule deviate from this bridge site slightly. The PP2 adsorption configuration is simply the PP1 configuration translated in the [001] direction by a distance equal to the half of the Ag lattice constant. For both configurations, the long molecular axis is not directly aligned along the [001] direction. The PP3 configuration is obtained by translating the PP1 configuration in the [110] direction by a distance equal to half of the nearest neighbor distance of $\mathrm{Ag}$. In this configuration, $\mathrm{S}$ atoms are positioned off center along [001] and [110] directions, and located over the top layer Ag atoms. Among all of the considered adsorption configurations of 6T adsorbed parallel to the [001] direction, namely PP3, gives the highest adsorption energy obtained using any of the functionals. The adsorption energy associated with the PP3 configuration differs from those of PP1 and PP2 by about $0.39 \mathrm{eV}$ and $0.54 \mathrm{eV}$ using the PBE functional; $1.02 \mathrm{eV}$ and $1.02 \mathrm{eV}$ using optB86b-vdW; $0.88 \mathrm{eV}$ and $0.96 \mathrm{eV}$ with optB88-vdW; $0.62 \mathrm{eV}$ and $0.68 \mathrm{eV}$ using optPBE-vdW; $0.02 \mathrm{eV}$ for revPBE-vdW, and $0.41 \mathrm{eV}$ and $0.45 \mathrm{eV}$ using rPW86-vdW2 functionals.

In Sec. III B, we discuss the adsorption heights of the 6T molecule on $\mathrm{Ag}(110)$ obtained using all of the vdW functionals considered here and compare them to the results obtained using PBE.

\section{B. 6T Adsorption heights on $\mathrm{Ag}(110)$}

The 6T adsorption heights on $\mathrm{Ag}(110)$, for all configurations, obtained using all the vdW functionals and $\mathrm{PBE}$, are summarized in Table I. Note that the adsorption height is defined in three ways in the Table I in order to determine any structural change induced in the molecule upon adsorption. The adsorption height $\left(\mathrm{d}_{\mathrm{ads} \_ \text {av }}\right)$ is obtained using the averaged $\mathrm{z}$ position differences between the atoms $(\mathrm{C}, \mathrm{H}$, and $\mathrm{S})$ in the 6T molecule and the averaged $\mathrm{z}$ positions of the $\mathrm{Ag}$ atoms in the first layer, while $\mathrm{d}_{\text {ads_CM }}$ and $\mathrm{d}_{\mathrm{SM}}$ are calculated using the difference in the averaged $\mathrm{z}$ positions of $\mathrm{C}$ and $\mathrm{S}$ atoms to those of the surface atoms, respectively. The comparisons between the adsorption heights suggest that the 6T molecule does not undergo any structural change upon adsorption, and that there is not any significant bending and/or tilting of the molecule when it is on the surface as shown in Figure 1(f), which shows the side views of the optimized 6T on $\operatorname{Ag}(110)$ for the two lowest energy configurations.

The overall trend of the changes in the adsorption heights with the vdW functionals is very similar to those obtained in recent studies for other systems on compact surfaces. ${ }^{32-35}$ The first thing that we notice is the reduction in the adsorption heights from those of PBE for all the configurations upon inclusion of $\mathrm{vdW}$ interactions via the opt-type functionals. The 6T molecule adsorbs closer to the Ag surface when using the opt-type functionals. The smallest adsorption heights were obtained using optB86b-vdW, followed by optB88-vdW, optPBE-vdW, rPW86-vdW2, and revPBE-vdW functionals.

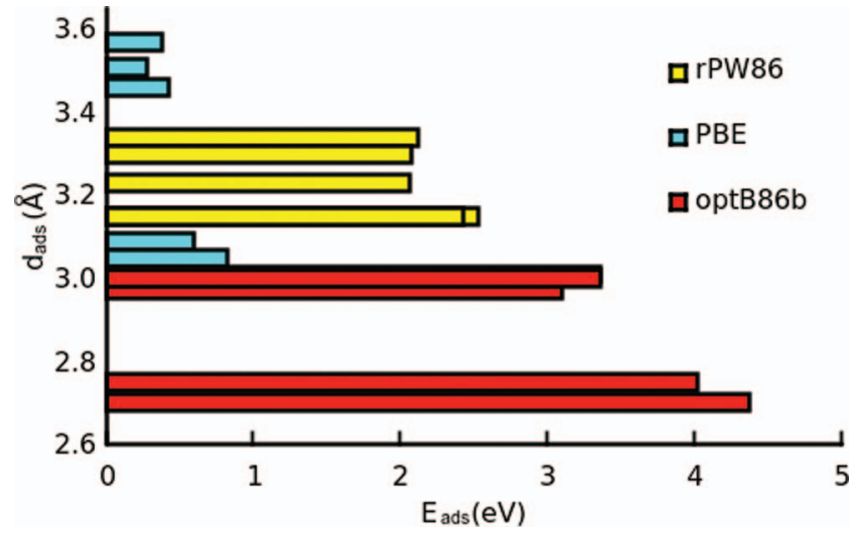

FIG. 3. 6T adsorption energies for all configurations as a function of adsorption heights on $\mathrm{Ag}(110)$ calculated using PBE, rPW86-vdW2, and optB86bvdW functionals. The results obtained using revPBE-vdW, optB88-vdW, and optPBE-vdW show similar trends and they are omitted for clarity (see Table I for comparison).

The 6T adsorption heights for the PL1 and PP3 configurations are smaller than those for the rest of the configurations; PP3 gives the shortest adsorption heights $(2.7 \AA)$ among all. The adsorption heights obtained using the opt-type functionals for the other configurations are between 3 and $3.2 \AA$, which are reduced from the range of 3.5-3.6 A obtained using PBE. The adsorption heights calculated for all the configurations using the revPBE-vdW and rPW86-vdW2 functionals are found to be higher than those of the opt-type functionals, and very similar or even higher (for PL1 and PP3) than those obtained with PBE. This is an expected observation, as these functionals are known to lead strong repulsion in general, and predict large adsorption heights. ${ }^{24,39,50,51}$ This was also the trend found in our earlier studies. ${ }^{32,33}$

We do not find any significant change in the height of the top layer Ag atoms upon adsorption of the 6T molecule. On average, using the optB86b-vdW functional, the interlayer separation was slightly less contracted with the adsorption of the 6T molecule as compared with the case at which the surface was bare. This is also the case using PBE although to a lesser degree. We attribute this to a stronger interaction between the molecule and the Ag substrate that allows the Ag surface atoms to relax toward the 6T molecule. The opposite effect is observed for the rPW86-vdW2 functional, for instance, that exhibits strong repulsion at small distances.

In Figure 3, we plot the adsorption energies as a function of adsorption heights for all configurations using the PBE, optB86b-vdW, and rPW86-vdW2 functionals. The figure shows the adsorption energy and height relation, and how it varies with the specific vdW functional used. For clarity in presentation, we choose only three functionals to illustrate our point, and note that other vdW functionals of the same group show a similar trend. The relation between the adsorption energies and heights are evident from the figure. The results obtained using PBE show lower adsorption energies than those obtained using the rPW86-vdW2 and optB86b-vdW functionals. Meanwhile, the adsorption heights are smaller with the optB86b-vdW functional and larger for rPW86-vdW2 functional than those of PBE. The substantial 
reduction in $6 \mathrm{~T}$ adsorption heights on $\operatorname{Ag}(110)$, obtained using the optB86b-vdW functional, may be correlated with the increase in adsorption energies. This functional is also shown to reproduce the highly nontrivial adsorption behavior of graphene on $\mathrm{Ni}$, as given by Random Phase Approximation (RPA) ${ }^{52}$ We reported a similar finding in our earlier study on benzene adsorption on metal surfaces ${ }^{32}$ for the opt-type functionals, which in general leads to a small adsorption distance to the surface of noble metal substrates. It is clear that the inclusion of vdW interactions, using the opt-type functionals, enhances molecule-surface interaction, increases the adsorption strength, and lowers the adsorption height. On the other hand, we find for the revPBE-vdW and rPW86-vdW2 functionals that although the adsorption energies are enhanced compared to those obtained using PBE, the adsorption heights are larger than those of PBE and the opt-type functionals. This observation once again suggests that the revPBE-vdW and rPW86-vdW2 functionals lead to repulsion upon adsorption of $6 \mathrm{~T}$. In comparison to the changes observed in adsorption heights for benzene using these functionals, we find that the current case deviates even further from the PBE results. This may be the effect of factors such as molecule size, the presence of $\mathrm{S}$ in the molecule, and adsorption on a more open surface with a different surface coordination. Note that the performance of these vdW functionals for functionalized organic molecule adsorption on metal substrates, as well as for adsorption on more open surfaces should be tested. Further systematic investigations are currently underway to assess the contribution of each effect.

\section{Changes in electronic structure upon $6 \mathrm{~T}$ adsorption on $\mathrm{Ag}(110)$}

In this section we examine the changes introduced to the electronic structure of the $\operatorname{Ag}(110)$ surface upon adsorption of the $6 \mathrm{~T}$ molecule. We first analyzed the changes in the position of the surface d-band centers $\left(\Delta \mathrm{E}_{\mathrm{d}}\right)$ and d-band widths $\left(\Delta \mathrm{W}_{\mathrm{d}}\right)$ upon adsorption. Most often the changes in the d-band features are used to explore the effect of adsorbed species on the host electronic structure and to help in classifying physisorbed/chemisorbed-bonding nature. ${ }^{53}$ This classification in turn informs on the possible changes in the electronic structure that will dictate the energy level alignments obtained when the bonding has a chemisorption character. The changes in the position of the surface d-band centers and d-band widths are summarized in Table II. The results suggest that the positions of the surface d-band centers shift toward lower binding energies (toward the Fermi level) when revPBE-vdW and rPW86-vdW2 functionals are used, while they shift toward higher binding energies for the opt-type functionals as compared with those obtained using PBE. The shifts in the positions of the d-band center upon the molecule's adsorption are the highest with the opt-type functionals; they are smaller and in the opposite direction with the revPBE-vdW and rPW86-vdW2 functionals. This can be correlated with the changes in the adsorption heights induced by these functionals. The opt-type functionals lower the adsorption heights from those of PBE, increasing molecule-substrate interaction leading to a shift toward higher binding energies.
TABLE II. Changes in the positions of the center of the d-bands, the dband widths, and the work functions upon $6 \mathrm{~T}$ adsorption calculated with varying vdW functionals for the two lowest adsorption energy configurations (PL1 and PP3) for 6T on $\operatorname{Ag}(110) . \Delta \mathrm{E}_{\mathrm{d}}$ and $\Delta \mathrm{W}_{\mathrm{d}}$ is obtained by subtracting the averaged d-band center positions and widths of the 6T on $\mathrm{Ag}(110)$ system from those of the clean $\operatorname{Ag}(110)$. The work function change is determined as $\Delta \Phi=\Phi^{6 \mathrm{~T} / \mathrm{Ag}(110)}-\Phi^{\mathrm{Ag}(110)}$. Negative sign indicates the reduction in the work function from the bare surface. The interface dipole measured by Grobosch et al. ${ }^{11}$ for high coverage 6T/Ag is shown here for comparison.

\begin{tabular}{lcccc}
\hline \hline Method & Configuration & $\Delta \mathrm{E}_{\mathrm{d}}(\mathrm{eV})$ & $\Delta \mathrm{W}_{\mathrm{d}}(\mathrm{eV})$ & $\Delta \Phi(\mathrm{eV})$ \\
\hline PBE & PL1 & -0.06 & 0.08 & -0.47 \\
optB86b-vdW & PP3 & -0.06 & 0.09 & -0.37 \\
& PL1 & -0.15 & 0.18 & -0.55 \\
optB88-vdW & PP3 & -0.12 & 0.18 & -0.48 \\
& PL1 & -0.11 & 0.19 & -0.56 \\
optPBE-vdW & PP3 & -0.12 & 0.17 & -0.51 \\
& PL1 & -0.09 & 0.10 & -0.47 \\
revPBE-vdW & PP3 & -0.09 & 0.12 & -0.44 \\
& PL1 & -0.04 & 0.07 & -0.34 \\
rPW86-vdW2 & PP3 & -0.08 & 0.08 & -0.25 \\
& PL1 & -0.07 & 0.08 & -0.46 \\
Experiment ${ }^{11}$ & PP3 & -0.06 & 0.07 & -0.38 \\
\hline \hline
\end{tabular}

On the other hand, the revPBE-vdW and rPW86-vdW2 functionals, due to their strong repulsive nature, the $6 \mathrm{~T}$ molecule adsorbs at a higher distance from the surface and the interaction is less strong with the substrate. Thus, a shift towards lower binding energies (towards Fermi level) is observed. The largest change in the position of the d-band center is in the order of $\sim 150 \mathrm{meV}$. The d-band widths show a slight variation upon adsorption. The results suggest that the d-band width broadens for the opt-type functionals and narrows for the revPBE-vdW and rPW86-vdW2 functionals, as compared to those obtained using PBE. This relates to the differences in the lattice constants of the surface obtained with different vdW functionals. Overall, the shifts in the positions of the dband centers and d-band widths are modest (60-190 meV), and they are not accompanied by the formation of interface states or any significant charge transfer (see below) to accommodate a chemisorbed bonding nature.

The change in the surface work function upon adsorption is often utilized to discuss the formation of an interface dipole. In earlier experimental studies, interfacial electronic structure properties have been reported for 6T adsorption on metal surfaces. An UV experiment reported an interface dipole of $0.7 \mathrm{eV}$, for $6 \mathrm{~T}$ adsorbed on polycrystalline $\mathrm{Ag},{ }^{11}$ which was determined from the reduction of work function upon adsorption. This study suggested that the reduction in the work function originates from the formation of an interface dipole as well as from the chemical interaction between the molecule and the substrate. Another study of $6 \mathrm{~T}$ on $\operatorname{Ag}(110)$, utilizing PEEM, measured an increase in the emission probability of $\mathrm{Ag}$ during the deposition of a $6 \mathrm{~T}$ monolayer that they attributed to a reduction in the work function of the system. ${ }^{12}$ Additionally, the UPS and XPS ${ }^{13}$ investigation, by Amsalem et al., reported charge transfer between $\mathrm{C}_{60}$ and a thin layer of $6 \mathrm{~T} / \mathrm{Ag}(111)$ substrate, and the formation of interface dipoles. 
We report, in Table II, the work function change $(\Delta \Phi)$ upon 6T adsorption on the $\operatorname{Ag}(110)$ surface using all the vdW functionals and PBE for the lowest adsorption energy configurations PL1 and PP3. Our results show that upon 6T adsorption, the work function decreases as compared with that of the bare $\operatorname{Ag}(110)$ surface. The decrease in the work function overall is the highest when using the opt-type functionals, in particular, the largest decrease is found for the opttype functionals, in the order of $\sim 0.48-0.56 \mathrm{eV}$. The reduction is found to be smaller for the rPW86-vdW2 and revPBE-vdW functionals when compared with the results of the opt-type functionals, however, found similar when compared to $\Delta \Phi$ using PBE. The work function decrease for the PL1 configuration is found to be slightly higher than that of PP3 for all functionals. This may be attributed to the differences in the coverage as our model system for PL1 configuration consists of a supercell of $4 \times 7 \mathrm{Ag}$ atoms per layer, while for PP3, the supercell is made of $2 \times 10 \mathrm{Ag}$ atoms per layer. Note that all the functionals yield a reduction in the work function, while those obtained using the opt-type functionals are close to but lower than the observed value of $0.7 \mathrm{eV} .{ }^{11}$ This is due to the fact that in our calculations, the coverage is always less than a monolayer, while the measurements were done at one monolayer and higher. For the PL configuration, the coverage is about 0.5 and that corresponding to the PP configuration is about 0.35 . Using these two coverages and their corresponding work function values, a linear extrapolation yields a value very close to the experimental value of $0.7 \mathrm{eV}$, when using the opt-type functionals.

Finally, we also performed Bader analysis to evaluate the charge transfer between the 6T molecule and the $\mathrm{Ag}(110)$ surface for all adsorption configurations using the vdW functionals and PBE. The charge transfer between the substrate and the molecule was determined to be smaller than 0.1e. Thus, our results suggest that, for close to a single layer $6 \mathrm{~T}$ adsorption on $\operatorname{Ag}(110)$, no significant charge transfer occurs between the molecule and the Ag surface. Additionally, the analysis of the total and partial electronic densities of states $\left(\mathrm{dz}^{2}\right)$ of the Ag surface atoms, before and after adsorption of $6 \mathrm{~T}$, shows no interface state formation. With the inclusion of $\mathrm{vdW}$ interactions, our results show significant change in the adsorption energies with reduction in the adsorption heights, no significant change in the electronic structure, no interface state formation, and negligible charge transfer. In the light of these results, we conclude that $6 \mathrm{~T}$ adsorption on $\mathrm{Ag}(110)$ can be characterized by strong physisorption as the minimal indications for a chemisorbed bonding nature are lacking.

\section{CONCLUSION}

The role of $\mathrm{vdW}$ interactions and their performance on the adsorption characteristics of $6 \mathrm{~T}$ on $\mathrm{Ag}(110)$ are studied using dispersion corrected density functional theory. Our calculations suggest that the lowest energy configuration of $6 \mathrm{~T}$ on $\mathrm{Ag}(110)$ corresponds to the case at which 6T lies with its long axis parallel to the [001] direction of $\operatorname{Ag}(110)$ and the next lowest energy configuration is that with the long axis perpendicular to the [001] direction. We find a small difference in the adsorption energy of these two configurations implying that they can co-exist, in agreement with the experimental observations. The inclusion of $\mathrm{vdW}$ interactions is found to enhance adsorption energies of $6 \mathrm{~T}$ as well as lower the adsorption heights for some $\mathrm{vdW}$ functionals. The largest changes in the adsorption energies and heights from those of PBE results are obtained with the opt-type functionals for which the molecule adsorbs closer and interacts more strongly with the substrate than those obtained using the PBE, revPBE-vdW, and rPW86-vdW2 functionals. The smaller increases in adsorption energies and larger adsorption heights are found using these functionals, as compared to the PBE results, and this is attributed to the strong repulsive nature of these functionals, as shown for few systems earlier.

For all adsorption configurations, however, no appreciable changes in the structures of both the molecule and the support are found upon adsorption. Furthermore, the analysis of the charge transfer between $6 \mathrm{~T}$ and the $\operatorname{Ag}(110)$ surface suggests that there is negligible charge transfer - for most cases below $0.1 e$. The changes in the electronic structure features of the Ag substrate are also found to be minimal upon adsorption. Furthermore, the shifts in the positions of the centers of the d-bands vary from $0.06 \mathrm{eV}$ to $0.15 \mathrm{eV}$ depending on the functional used, and the maximum change in the d-band widths is $0.17-0.19 \mathrm{eV}$, using the optB88-vdW and optB86b$\mathrm{vdW}$ functionals. Upon adsorption, we find that the surface work function is reduced, and that the largest reduction in the work function, of $0.56 \mathrm{eV}$, results with the optB88-vdW functional. Our result is in good agreement with the experimental observations for the reduction of the work function introduced by $6 \mathrm{~T}$ adsorption.

Based on the results obtained here, namely, the small changes in the atomic and electronic structures of the substrate and 6T molecule, negligible charge transfer, and no interface state formation upon adsorption of the molecule, we conclude that the nature of bonding for 6T adsorption on $\operatorname{Ag}(110)$ can be classified as strong physisorption.

\section{ACKNOWLEDGMENTS}

We acknowledge support from the U.S. Department of Energy Basic Energy Science under Contract No. DE-FG0211ER16243. This research used resources of the National Energy Research Scientific Computing Center, which is supported by the Office of Science of the U.S. Department of Energy under Contract No. DE-FG02-11ER16243.

${ }^{1}$ G. Horowitz, J. Mater. Res 19, 1946 (2004).

${ }^{2}$ Z. Bao, Adv. Mater. 12, 227 (2000).

${ }^{3}$ Y.-Y. Lin, D. Gundlach, S. Nelson, and T. Jackson, IEEE Electron Device Lett. 18, 606 (1997).

${ }^{4}$ R. Pande, S. Kamtekar, M. S. Ayyagari, M. Kamath, K. A. Marx, J. Kumar, S. K. Tripathy, and D. L. Kaplan, Bioconjugate Chem. 7, 159 (1996).

${ }^{5}$ M. Şenel, M. Dervisevic, and E. Çevik, Curr. Appl. Phys. 13, 1199 (2013).

${ }_{6}^{6}$ I. F. Perepichka, D. F. Perepichka, H. Meng, and F. Wudl, Adv. Mater. 17, 2281 (2005).

${ }^{7}$ N. Noma, T. Tsuzuki, and Y. Shirota, Adv. Mater. 7, 647 (1995).

${ }^{8}$ B. S. Ong, Y. Wu, P. Liu, and S. Gardner, J. Am. Chem. Soc. 126, 3378 (2004).

${ }^{9}$ G. Yoshikawa, M. Kiguchi, S. Ikeda, and K. Saiki, Surf. Sci. 559, 77 (2004). 
${ }^{10}$ T. Schwieger, X. Liu, H. Peisert, B. Adolphi, N. Kiriy, and M. Knupfer, J. Appl. Phys. 97, 123712 (2005).

${ }^{11}$ M. Grobosch and M. Knupfer, Org. Electron. 8, 625 (2007).

${ }^{12}$ T. Wagner, D. R. Fritz, and P. Zeppenfeld, Synth. Met. 161, 2006 (2011).

${ }^{13}$ P. Amsalem, J. Niederhausen, A. Wilke, G. Heimel, R. Schlesinger, S. Winkler, A. Vollmer, J. Rabe, and N. Koch, Phys. Rev. B 87, 035440 (2013).

${ }^{14}$ S. Gottardi, K. Müller, J. C. Moreno-López, H. Yildirim, U. Meinhardt, M. Kivala, A. Kara, and M. Stöhr, Adv. Mater. Interfaces 1, 1300025 (published online, 2013).

${ }^{15}$ K. Müller, A. Kara, T. K. Kim, R. Bertschinger, A. Scheybal, J. Osterwalder, and T. A. Jung, Phys. Rev. B 79, 245421 (2009).

${ }^{16}$ M. Kiguchi, S. Entani, K. Saiki, and G. Yoshikawa, Appl. Phys. Lett. 84, 3444 (2004).

${ }^{17}$ S. Prato, L. Floreano, D. Cvetko, V. D. Renzi, A. Morgante, S. Modesti, F. Biscarini, R. Zamboni, and C. Taliani, J. Phys. Chem. B 103, 7788 (1999).

${ }^{18}$ P. Hohenberg, Phys. Rev. 136, B864 (1964).

${ }^{19}$ W. Kohn and L. J. Sham, Phys. Rev. 140, A1133 (1965).

${ }^{20}$ K. Müller, A. P. Seitsonen, T. Brugger, J. Westover, T. Greber, T. Jung, and A. Kara, J. Phys. Chem. C 116, 23465 (2012).

${ }^{21}$ M. Dion, H. Rydberg, E. Schröder, D. C. Langreth, and B. I. Lundqvist, Phys. Rev. Lett. 92, 246401 (2004).

${ }^{22}$ T. Björkman, A. Gulans, A. Krasheninnikov, and R. Nieminen, J. Phys.: Condens. Matter 24, 424218 (2012).

${ }^{23}$ F. Furche, J. Chem. Phys. 129, 114105 (2008).

${ }^{24}$ J. Klimeš, D. R. Bowler, and A. Michaelides, J. Phys.: Condens. Matter 22, 022201 (2010).

${ }^{25}$ A. Tkatchenko, L. Romaner, O. T. Hofmann, E. Zojer, C. Ambrosch-Draxl, and M. Scheffler, MRS Bull. 35, 435 (2010).

${ }^{26}$ J. Carrasco, B. Santra, J. Klimeš, and A. Michaelides, Phys. Rev. Lett. 106, 026101 (2011).

${ }^{27}$ V. G. Ruiz, W. Liu, E. Zojer, M. Scheffler, and A. Tkatchenko, Phys. Rev. Lett. 108, 146103 (2012).

${ }^{28}$ S. Grimme, Wiley Interdiscip. Rev.: Comput. Mol. Sci. 1, 211 (2011).

${ }^{29}$ K. Burke, J. Chem. Phys. 136, 150901 (2012).

${ }^{30}$ C. D. Sherrill, J. Chem. Phys. 132, 110902 (2010).
${ }^{31}$ K. E. Riley, M. Pitoňák, P. Jurečka, and P. Hobza, Chem. Rev. 110, 5023 (2010).

${ }^{32}$ H. Yildirim, T. Greber, and A. Kara, J. Phys. Chem. C 117, 20572 (2013).

${ }^{33}$ H. Yildirim and A. Kara, J. Phys. Chem. C 117, 2893 (2013).

${ }^{34}$ J. Carrasco, J. i. Klimeš, and A. Michaelides, J. Chem. Phys. 138, 024708 (2013).

${ }^{35}$ W. Liu, J. Carrasco, B. Santra, A. Michaelides, M. Scheffler, and A. Tkatchenko, Phys. Rev. B 86, 245405 (2012).

${ }^{36}$ J. P. Perdew, K. Burke, and M. Ernzerhof, Phys. Rev. Lett. 77, 3865 (1996).

${ }^{37}$ J. P. Perdew, M. Ernzerhof, and K. Burke, J. Chem. Phys. 105, 9982 (1996).

${ }^{38}$ J. Klimeš and A. Michaelides, J. Chem. Phys. 137, 120901 (2012).

${ }^{39}$ J. Klimeš, D. R. Bowler, and A. Michaelides, Phys. Rev. B 83, 195131 (2011).

${ }^{40}$ T. Thonhauser, V. R. Cooper, S. Li, A. Puzder, P. Hyldgaard, and D. C. Langreth, Phys. Rev. B 76, 125112 (2007).

${ }^{41}$ E. D. Murray, K. Lee, and D. C. Langreth, J. Chem. Theory Comput. 5, 2754 (2009).

${ }^{42}$ K. Lee, É. D. Murray, L. Kong, B. I. Lundqvist, and D. C. Langreth, Phys. Rev. B 82, 081101 (2010).

${ }^{43}$ G. Kresse, Phys. Rev. B 54, 11169 (1996).

${ }^{44}$ G. Kresse and J. Furthmüller, Comput. Mater. Sci. 6, 15 (1996).

${ }^{45}$ G. Kresse and J. Hafner, Phys. Rev. B 47, 558 (1993).

${ }^{46}$ G. Kresse, Phys. Rev. B 59, 1758 (1999).

${ }^{47}$ P. E. Blöchl, Phys. Rev. B 50, 17953 (1994).

${ }^{48} \mathrm{R}$. Bader, Atoms in Molecules: A Quantum Theory (Clarendon, Oxford, UK, 1990).

${ }^{49}$ W. Tang, E. Sanville, and G. Henkelman, J. Phys.: Condens. Matter 21, 084204 (2009).

${ }^{50} \mathrm{~J}$. Wellendorff and T. Bligaard, Top. Catal. 54, 1143 (2011).

${ }^{51}$ V. R. Cooper, Phys. Rev. B 81, 161104 (2010).

${ }^{52}$ F. Mittendorfer, A. Garhofer, J. Redinger, J. Klimeš, J. Harl, and G. Kresse, Phys. Rev. B 84, 201401 (2011).

${ }^{53}$ A. K. Kelkkanen, B. I. Lundqvist, and J. K. Nørskov, Phys. Rev. B 83, 113401 (2011). 